Peter Kirwan

University of Warwick

\title{
"What's Past is Prologue": Negotiating the Authority of Tense in Reviewing Shakespeare
}

Since 2006, when it was set up as my postgraduate project to chronicle the Royal Shakespeare Company's Complete Works Festival, the Bardathon review blog has been experimenting with reviewing technique. Taking advantage of the discursive and instantaneous platform that blogging offers, while remaining committed to the longerfocus descriptive and analytic emphasis of the academic review, the blog aims to stimulate academic discussion in the immediate aftermath of performance.

The most basic practical consideration that arises in bringing together the two forms of review is the selection of tense. The journalistic review is almost invariably written in the present tense, while the academic review, usually written and published months after the production has closed, speaks of the production in the past. This is generally, and unconsciously, a reflection of how closely the act of writing occurs after the act of viewing. When, however, is the line crossed? When does a production or performance move into the past? These questions are more than merely semantic. This paper argues that the choice of reviewing tense carries significant interpretative implications for the object of review, the purpose of review, and the reviewer themselves.

\section{Production or performance?}

In arts journalism, the theatre review presents a grammatical anomaly. Elsewhere, present tense is used for the durable artwork or event: a film, a record, an exhibition, something that may be re-experienced in the same form as that reviewed. By contrast, the 
Peter Kirwan

University of Warwick

past tense is generally reserved for live events: rock concerts, sports matches. These events are regarded as involving an amount of spontaneity and unpredictability, making the event a "one-off" that may not be re-experienced. Consequently, by writing in the present tense, the theatrical reviewer inferentially treats live performance as unchanging and constant, a durable phenomenon that the reader may experience in the same form. It exists; it continues to exist.

The use of present tense thus blurs the distinction between production and performance, between the ongoing run of performances and the specific occasion attended by the reviewer. A moment of performance is taken to represent all potential moments of performance. This is an accepted part of contemporary theatrical culture, with the press night carefully timed to minimise the risk of variation, normally occurring late enough in the run that the production is felt to have settled, but early enough that good notices will serve to boost ticket sales. This performance is authorised as the "definitive" version for the purposes of the reviewer. The present tense reinforces the collective agreement that the selected performance is the moment for canonisation. This performance "is" - and continues to "be" - the production, a single incarnation preserved for posterity.

For archival purposes, this poses immediate problems, as the institutionallyapproved performance is the only one recorded by reviews, even if later performances have incorporated new adjustments or improvements. The established convention that the press performance is the single-moment embodiment of a production's entire run has been further challenged in recent years by the advent of blogging and other media, allowing amateur and professional critics to post reviews of non-press night performances for a worldwide online audience. This democratisation of performance criticism has gone some 
Peter Kirwan

University of Warwick

way towards diminishing the importance of press nights, introducing a "mystery shopper" atmosphere in which any performance may be reviewed. However, regardless of the date of performance, the use of the present tense continues to perform the function of extrapolating from a single performance what a production did, does and continues to do.

The limitations of this are highlighted by those occasions where the unexpected occurs. During the press night of the RSC's 2006 Antony and Cleopatra, a fire alarm caused a full evacuation of the Swan, an occurrence remarked upon by most broadsheet reviews. All of their reviews were subsequently written in the present tense except for those remarks on the evacuation, for which they reverted to the past tense. ${ }^{1}$ In this instance, it was understood that the fire alarm was not a planned part of the evening, and thus it was accorded the past-tense treatment usually reserved for live events.

Tense is dictated by the reviewer's perception of intentionality. If something was meant to happen, the present tense indicates this and canonises the decision; if it was not meant to happen, the past tense positions it as a fluke. However, the reviewer is unqualified to judge a production's intentionality. An actor's accidental omission of a line can be read by a reviewer as a director's deliberate cutting of the text, but for the reviewer to write his response in the present tense memorialises a decision that was as unique to the moment of performance as a fire alarm. The usefulness of the reviewer, as a critical spectator, comes not in evaluating what a performance meant to do (for which there are other resources: prompt books, director interviews) but for what it did do.

By establishing the object of review as the one-off performance rather than the production, the authority of the reviewer is consequently defined within clear temporal

\footnotetext{
${ }^{1}$ See, for example, Paul Taylor, Independent, 21 April 2006; Benedict Nightingale, Times, 21 April 2006; and Charles Spencer, Telegraph, 20 April 2006.
} 
Peter Kirwan

University of Warwick

parameters. Reviewing a performance rather than a production releases the reviewer from the need to extrapolate intentionality from a live event. A reviewer's authority is rooted in the individual experience of the specific moment, the view from their seat. By locating the object of review as a past, defined event, the reviewer can assume authority, speaking not of what should or was meant to happen, but what did happen. In turn, this allows the review to take into account a performance's spontaneity and immediate context, prioritising a real event over an imagined - and incomplete - full theatrical run.

\section{Preserving or selling?}

The question of whether a review refers to performance or production has further implications for the purpose and scope of the review. The present tense implies an authority free of temporal constraints and acts as a promise: this is what happens, this is what you will experience. The present is continuous and serves to cover the production's future, with the reviewing voice speaking authoritatively on intentionality as pertaining to the remainder of the production's run. The past tense, however, acts with the authority of the archive: this is what happened, this is what was experienced. Both are authoritative, but serve different purposes: the one serves to articulate and shape the immediate and future response to a production, while the other serves to catalogue and position the production historically.

It is primarily for this reason that academic and journalistic reviews differ in their use of tense. Newspapers respond to and document the present (and, implicitly, the future), while academic archives and journals organise and make sense of the past. With the advent 
Peter Kirwan

University of Warwick

of new reviewing platforms, however, a third space has emerged which may usefully be termed the "academic review blog". Projects such as Internet Shakespeare Editions, Hot Reviews and The Bardathon, whether collectively or individually authored, consciously serve dual purposes, responding immediately and subjectively to performance while using the longer discursive format to establish a valuable historical archive.

Most theatre blogging follows the journalistic model, recognising itself as essentially ephemeral and using the present tense. The academic blog, however, aims to operate as an immediate archive, and to do so must differentiate itself. The aim is for the reviews not to be transient - read today, forgotten tomorrow - but available for repeated viewing and as resource material, archiving the present response. Reviews are instantly catalogued and electronically filed, hyperlinked with others to build a coherent, permanent electronic resource. As such, it is necessary to identify the temporal moment and subjective viewpoint that the review preserves, positioning it precisely within the repository.

By writing the review in the past tense, even if the performance was viewed the day before, a different set of concerns emerges. The review no longer acts to promise or persuade, but to describe and discuss. Its scope is narrower, concerned with a single past moment of performance, but its authority is absolute within those defined limits: the reviewer speaks with unmatched authority on their own experience of a specific performance.

The present tense betrays a fundamental anxiety inherent in the promise it implies. It extrapolates the future from the present, entering into a dialogue with the responses of readers for whom the moment of performance has not yet even begun. It is, therefore, a promise that is open to being unfulfilled. In the event of the production evolving or 
Peter Kirwan

University of Warwick

changing, the present tense becomes outdated and, more damningly, incorrect. The promise of what the production "is" is proven false, at worst exposing the reviewer to charges of basic factual inaccuracy, at best demonstrating the insufficiency of the reviewer's experience. In this sense, the present tense renders the reviewer vulnerable. Its implied universality and timelessness create an impression of continuous authority that is easily undermined by changes to the production that render the reviewer's facts retrospectively incorrect - and a reader has no reason to take heed of a review that factually fails to match their experience. The only defence the reviewer has against charges of incorrectness in this situation is to resituate the reviewer's experience temporally: this is what I saw, this is what the production did do, thus reasserting the reviewer's unimpeachable authority to report on their own experience. At the performances I attended of the RSC's 2006 Julius Caesar and Toneelgroep Amsterdam's 2009 Roman Tragedies, the actors playing Mark Antony (Ariyon Bakare and Hans Kesting respectively) were confined to crutches or wheelchair following accidents. While this was unplanned in both cases, I found Bakare's war-wounded, limping Antony to be a dangerous threat entirely underestimated by the conspirators. Kesting's wheelchair-bound Antony, meanwhile, manipulated the condescending attitudes of the other politicians to be allowed to give his funeral oration. These performances were aberrations from the intended production, and to universalise them in the present tense would be misleading in the context of the play's international performance history; yet they were still as much a part of this reviewer's experience as other aspects of the production. Through reviewing the productions in the past tense, the uniqueness of the experience could be noted and made 
Peter Kirwan

University of Warwick

available for comparison with the experience of others who saw the performances "as intended", a record of a moment rather than a promise for the future.

\section{Human or god?}

The final implication of tense is in negotiating the persona of the reviewer. The use of the present tense, in the journalistic or electronic environment of immediate response and publication, articulates the reviewer's thoughts at the moment of writing; they represent the contextually-situated "now" of reviewer, production, culture and medium. In the archive, however, the present tense denies and obscures the reviewer's own development. Two different studies from the "Shakespeare in Performance" series provide instructive examples. Margaret Shewring's Richard II (1996) adheres to the past tense throughout in describing historical performances of the play as late as 1991. In her afterword, however, she notes that "As this study goes to press, the staging of Richard II is once again at the centre of critical attention. A compelling new production opened on 2 June 1995" before switching to present tense for her analysis: "the production challenges previous approaches..." (180-1). Here, tense is used consciously to articulate an important division between the stage history Shewring has written, and the stage future that is yet to be fully absorbed. The author is precisely temporally located; this is a history of the play as perceived from this point in time. By contrast, James Bulman's The Merchant of Venice (1991) discusses (or, indeed, discussed) several major productions of the play between 1879 and 1987, using the past tense to speak of those productions that occurred before his time, and the present for those that Bulman personally saw between 1970 and 1987 . The 
Peter Kirwan

University of Warwick

study thus conflates a generation of theatregoing into a single, imagined present, what

David Roberts refers to as the "golden realm of the now" (349).

There are two key difficulties with Bulman's use of tense. Firstly, the long span of his present tense turns this reviewer into an omniscient figure, capable of discussing in the immediate moment events that occurred eighteen years apart. Laurence Olivier's 1970 Shylock becomes a contemporary of Antony Sher's in 1987, and the contextual circumstances surrounding the two collide in the reader's mind. The distinction between those productions described in past or present is meaningless for the reader: the productions of Henry Irving and Theodore Komisarjevsky are past, the productions of Jonathan Miller and Bill Alexander are present, current. The question with which I began When does a production move into the past? - is, in Bulman's case, simply a matter of the reviewer's personal experience; yet it creates an artificial distinction in a continuous performance history that only has meaning for the reviewer. For Bulman, the "present" begins somewhere around the 1960s; yet for the reader in 2009, his "present" productions are already part of an increasingly distant past.

The second, related problem is to do with the reviewer's own development. Over an eighteen-year period, Bulman himself gained in experience, and the eyes with which he saw the 1970 Merchant were not the same as those with which he saw the 1987 production. A further four years had elapsed by 1991, the year of the volume's publication. Bulman begins his discussion of the Alexander production in the past tense, historically situating the production in light of the theatrical history to which he was responding: “Audiences were thus invited to cheer Portia's victory [...] Sher deliberately made Shylock offensive" (119). He then, without warning switches mid-paragraph to the present tense: 
Peter Kirwan

University of Warwick

"he is squatting cross-legged on a cushion [...] Sher uses his vocal limitations intelligently"

(121). The impression is that the reviewer is travelling back in his mind's eye, reliving the experience of viewing the production. The present tense acts as an enabling fiction that presents the response to stage action as immediate and unfiltered, imagining the reviewer as a two-way transparent conduit through which the reader might glimpse the performance. Here, however, the inconsistent switches in tense betray a conflation of perspectives that undermine this illusion and deny the writer's own experience and personal development.

These two examples highlight a fundamental but overlooked fact of reviewing: that the moment preserved is not the moment of seeing, but the moment of writing, which necessarily takes place some time after the performance, thereby immediately positioning the performance in the past. The present tense attempts to capture the experience of being in the auditorium, watching the production as a live event. However, unless we are posting our review via a live Twitter feed (as trialled by the West End Whingers for Trevor Nunn's 2008 Gone With the Wind), this is a fallacy. Our response to the production evolves with every minute that distances us from the performance, and even within the performance itself: the events of Act Two change our response to what we have seen in Act One, and so on. The act of reviewing (literally, of course, viewing back) is an act of remembering, and we remember the past, not the present.

For the review, then, the use of the past tense enables both the act of viewing and the act of reviewing to be temporally located in relation to one another. The relationship between performance and spectator does not necessarily end with the curtain call. In the act of writing and further re-viewing, the memory of the performance and the reviewer's 
Peter Kirwan

University of Warwick

response to it change. The present tense not only universalises the live performance, but ossifies the reviewer's opinion, forestalling the possibility that that opinion may continue to develop. By contrast, the past tense allows the review to act as a time capsule, capturing and archiving the moment of response, which can then be revisited and responded to in turn.

\section{Conclusions}

While the present tense continues to serve the immediate commercial demands of the journalistic review, it must be accepted that the role of the theatrical review is changing. The democratisation of critical opinion has provoked violent debates over the continued usefulness of the professional review in an environment where opinions come cheaply and amateur "vox pops" sit alongside press reviews on websites and marketing materials. The value of the professional opinion, therefore, may be more usefully located in its archival positioning, locating a production in both the history of the play and the history of the experienced reviewer.

If professional theatre criticism is therefore to rearticulate its authority, its "USP", it can utilise the past tense to do so, reconfiguring performance as live experience and the reviewer as the archivist of a moment. What the past tense loses in not simulating an omniscient, objective experience of an entire production, it gains in identifying a true, subjectively-realised moment over which the reviewer has absolute authority, communicating to the reader not clinical observations of a fixed object, but an engaged report of a lived and unrepeatable moment. 
Peter Kirwan

University of Warwick

\section{Works Cited}

Bulman, James C. The Merchant of Venice. Shakespeare in Performance. Manchester: Manchester University Press, 1991.

Nightingale, Benedict. "Antony and Cleopatra." Rev. of Antony and Cleopatra by the RSC. Times 21 Apr. 2006. Print.

Roberts, David. “Shakespeare, Theater Criticism, and the Acting Tradition." Shakespeare Quarterly 53 (2002): 341-61.

Shewring, Margaret. King Richard II. Shakespeare in Performance. Manchester: Manchester University Press, 1996.

Spencer, Charles. "Middle-Aged Passion Goes to the Heart." Rev. of Antony and Cleopatra by the RSC. Telegraph 20 Apr. 2006. Print.

Taylor, Paul. "She Stoops to Conquer." Rev. of Antony and Cleopatra by the RSC. Independent 21 Apr. 2006. Print.

The West End Whingers. "Review - Gone With The Wind - The Musical! (Act 2) at the New London Theatre." West End Whingers, 2008. Web. 2 Nov. 2009. 